\title{
RELIABILITY OF MOBILE APP ECOSYSTEM
}

\author{
Seung C. Lee, University of Minnesota at Duluth, slee@d.umn.edu
}

\begin{abstract}
Digital ecosystems such as app ecosystems are viewed as digital counterparts of biological ecosystems. As in biological ecosystems, the attributes of the members, such as diversity, play an important role in digital ecosystem functioning and reliability. In an app ecosystem, only a few apps are successful and the vast majority of apps do not succeed by any measure. This implies that most of the apps in an app ecosystem can be considered redundant. In this paper, I argue that we should value app redundancy and consider it to be a critical feature of an app ecosystem for the system to function reliably over a long period of time.
\end{abstract}

Keywords: App Ecosystem, App Redundancy, Ecosystem Functioning, Ecosystem Reliability

\section{INTRODUCTION}

A biological ecosystem is defined as a community of living organisms (biotic factors) considered together with its environment (abiotic factors) functioning as a system through complex interactions [15, 23, 76, 86]. A biotic factor is any living organism in a food chain of autotrophs and heterotrophs. They are producers, consumers and decomposers, which cannot be separated from abiotic factors [61, 76]. Abiotic factors are non-living components of a biotic component's environment, such as temperature, light, moisture, air currents, etc.

The nature of the biotic factors, such as the species, functional groups, and biodiversity, plays an important role in ecosystem functioning [73], where biodiversity or biological diversity refers to the levels of variability of the biotic factors, including diversity within species, between species, and of ecosystems [8]. The notion of functional groups refers to grouping species by ecological equivalency such as roles and behavior [69] and trophic levels [30]. Within the context of ecosystem functioning, functional groups can be defined as collections of species that share a common set of biological properties and behavior. Biodiversity of a functional group can be measured by species diversity that is made up of two components: species richness and species evenness. Species richness is the number of different species, whereas species evenness measures the abundance of a specific species [34, 81, 83].

A digital ecosystem is viewed as a digital counterpart of a biological ecosystem [10, 24]. It has been argued that the studies of biological ecosystem functions and functioning provide useful yet powerful guidelines and terms for understanding digital ecosystems [36, 54, 55, 64, 70]. A digital ecosystem is defined as an open, socio-technical system that resembles a biological ecosystem, which exhibits such characteristics as robustness, scalability, and selforganization that can be found in a biological ecosystem [44, 79]. A digital ecosystem can also be defined for different domains [14]. One of the important domain-specific digital ecosystems is mobile ecosystem where a large and complex network of members (actors or players) - from, to name a few, infrastructure providers, device manufacturers, platform providers, app developers, apps, app stores, payment gateway service providers to consumers - interact with each other, directly and indirectly, to provide a broad collection of ecosystem products and services to end-users [6]. Like in a biological ecosystem [45], the members in a mobile ecosystem can also functionally be grouped based on their attributes. Each functional group is, therefore, made up of functionally equivalent or similar members where the term members correspond to species in ecology. Although defining the precise boundaries of an ecosystem is impossible [36], we can even think of smaller ecosystems within a mobile ecosystem. Among them is the app ecosystem in which several key functional groups of a mobile ecosystem, such as platform providers, app developers, app stores, apps, and end-users, interact with each other [3,5,7,65]. Practically, the entire collection of apps for a mobile platform can be considered a functional group of the mobile app ecosystem, although the apps can further be classified into smaller categories or functional groups (e.g., [52]). The more the number of apps a mobile platform has, therefore, the higher the app diversity the mobile app ecosystem exhibits, just as the biodiversity in a biological ecosystem does. If the number of apps increases, the app diversity also increases. But what are the implications of higher app diversity for the mobile app ecosystem functioning? 
With over 1 million apps for the Android platform alone [43], it is obvious that app developers and stores need to expend more effort to stand out from the crowd. Although the number of downloads from the app stores topped 102 billion as of 2013 and is expected to reach 268 billion by 2017 [46], many apps are tried a few times and then discarded. Indeed, almost $22 \%$ of apps downloaded are uninstalled after just one time use [77]. This implies that a lot of apps that even enjoy higher number of downloads simply experience a higher churn rate without generating any substantial financial and other meaningful results. Even though there are still many successful app developers who made fortunes off the explosion in mobile apps, the success rate is almost infinitesimal [27]. Majority of the independent developers have failed and ended up realizing that coding skills backed by a good idea do not guarantee any meaningful success in the competitive market. With millions of apps already on the app stores, and tens of thousands more launched every month, many developers and startups are finding that their success is far from reality $[25,71]$.

This means that, in the context of financial success or by any measure, all apps are not created equal. At one end of the continuum some apps are determinants, or the drivers, of a functional group (e.g., a specific category of apps such as games) of the app ecosystem of which they form a part. At the other end are those that are the passengers. Yanking the former may bring up agitation and clamor among the consumers in the app ecosystem [26,60], or the ecosystem can be flooded with cloned apps [66], but loss of the passengers might have little impact on the app ecosystem. Apparently, some of the passengers might have used to be the drivers (e.g., [49]) or some of them might have been underdogs at the outset. In much the same vein, a few of the passengers could someday become the drivers for any reason such as changes in the app ecosystem's environment or by recalibration. Whatever lurks behind the passengers' misfortunes or whether they ever see the silver lining on the clouds, it appears that the passengers are superfluous for the app ecosystem. The question then arises, "Are the passengers or underperforming apps really redundant in its literal sense on the functioning and reliability of the app ecosystem?"

In this paper, I present a way of examining the relationship between app diversity and app ecosystem functioning and reliability. Higher app diversity, as in biological ecosystems [58, 84], results in higher app redundancy because all apps are not created equal in terms of financial or other aspects of success [27]. Therefore, assessing the relationship between app diversity and app ecosystem functioning and doing the same thing between app redundancy and app ecosystem functioning are two sides of the same coin. The proposed assessment method is based on the concepts from reliability engineering, where reliability refers to the ability of a system or component to perform its required functions under given conditions for a specified period of time [38, 89]. The next two sections discuss ecosystem functions, ecosystem functioning, and functional groups in an ecosystem from both perspectives of natural and business ecosystems. The section following them illustrates local diminution of ecosystem members, ecosystem complexity, as well as ecosystem failure from a reliability engineering perspective. The remaining sections presents app redundancy and app ecosystem reliability, and the value of app redundancy.

\section{ECOSYSTEM FUNCTIONS}

One of the interests in ecological studies has been around the term function as in the function of biodiversity, the analysis of ecosystem functioning, the idea of functional redundancy of species, and functional groups of species (e.g., $[9,45])$. The word function can be interpreted in four different perspectives. First, it refers to what happens between organisms in an ecosystem (e.g., a wolf eats a rabbit or nutrients in dead organic matter are decomposed and then reused by plants). That is, the term function is used to describe certain processes, interactions, or relationships among species [39]. If applied to an app ecosystem, the term function would mean the relationships among functional groups and their members. For example, platform providers make various APIs available to developers; they use the APIs to create apps and make them available on an app store; users download apps from the app store; some apps are more popular than others, and so on. The second definition of the word function is related to the role of an organism or a functional group (a group of species that share a common set of attributes) in making the whole ecosystem sustainable or functioning [39]. In the context of app ecosystem, this would be about the contributions each member or a functional group makes to the app ecosystem so that it can maintain a certain state or trajectory supported by the processes described before. For example, the platform architectures and control structures together determine the platform's evolutionary trajectory, which leads to platform differentiation, different app development process, and unique user experiences $[22,79]$.

The third perspective for the term function is related to the ecosystem members' multiplicity of roles. For example, a tree is not only an organism assimilating nutrients via photosynthesis, but also it is a primary producer within the 
ecosystem. This notion of function is useful in classifying members of an app ecosystem into functional groups, based on their roles or survival strategies in the system [87]. For example, consumer app developers can leverage their experiences and name recognition gained through the presence in the consumer-oriented app markets to become successful enterprise app developers [4]. Some users are also developers, too, as another example. Finally, the word function is viewed as a particular service of an ecosystem for humans because it produces various goods, such as oxygen, game, and forest products, for human well-being and consumption [51].

\section{Ecosystem Functioning and Functional Groups}

Although natural and business ecosystems share some key characteristics, including competition, cooperation, exploitation, and evolution [35, 70] beyond robustness, scalability, and self-organization, there are also some differences between natural and business ecosystems. [54, 55] suggest that members in the business ecosystem engage themselves in more conscious decision-making by understanding the variables and calculating alternatives, that is, business ecosystem members are intelligent agents capable of planning and organizing for growth, not just for survival [35]. In any case, focusing on the whole ecosystem relates to questions such as how ecosystem is maintained and functioning and which roles particular species or biodiversity in a biological ecosystem (for an app ecosystem, particular apps or app diversity) play for this functioning, or if there are species (or apps for an app ecosystem) which may be considered redundant [39]. Rather than considering the questions at the individual species level, however, many studies in ecology and conservation have been done at the level of functional groups of species (e.g., [85]).

For clarification, I define, based on the commonalities of the previous conceptualizations, a functional group, within the context of app ecosystem functioning, as a group of members that shares common socio-technical characteristics. However, a natural ecosystem can also be modelled by trophic components, such as producers, consumers, decomposers, and others, instead of functional groups [12]. These components are linked by the ecosystem life cycle phases. [32] identified six different phases or flows that link these components: acquisition of energy, mineralization, decomposition, immobilization, death, and assimilation. The six phases in the natural ecosystem life cycle corresponds, albeit loosely, to the phases of the industry life cycle [67] and its variants (e.g., [48]). Although both natural and business ecosystems have much more complex structures than the ones simplified by their functional groups and linked components, this basic understanding provides the basis for assessing redundancy and functioning in natural and business ecosystems, particularly in app ecosystem.

\section{Local Diminution, Ecosystem Complexity, and Ecosystem Failure}

Within a natural ecosystem, each species has a finite probability of a local diminution (or even extinction in some extreme cases), where local indicates a population of a specific species. Different species appear to have different probabilities of a local diminution (e.g., $[2,53,63]$ ). A local diminution can be caused by many different factors in a natural ecosystem, including unstable relationships with predators, extreme environmental conditions such as a drought or fire, population size, and lost habitat $[33,63]$. In a similar fashion, each member of a functional group in a digital ecosystem has a finite probability of failure. For example, each app in a certain category of apps (i.e., a functional group) in an app ecosystem has a finite probability of failure by any measure. Different apps, depending on various factors, are likely to have different probabilities of failure. We can think of many contributing factors that can affect the probability of failure (or success), such as a level of marketing, idea uniqueness, word-of-mouth, performance, functionality, usability, ease of use, level of challenge, user engagement and acceptance, fun, intriguing, being informative, target audience, price, in-app purchase, expandability, network externality, and so on [20, 40, 65, 71]. Hence, it can be said that failure of an app within a functional group of an app ecosystem is a stochastic process, not a deterministic process, because no one knows if an app is a failure until it actually fails.

\section{Ecosystem Complexity}

In the complex systems theory, the term complexity is defined as an entity with many dimensions in which multiple components interact with each other in various ways [88]. Just as the complexity of a natural ecosystem can be defined in terms of multiple dimensions [11], the complexity of a business ecosystem can be viewed in many different perspectives. For example, [6] identified convergence and its consequences (e.g., elevated competition) as key factors that have increased the level of complexity of the network of members or actors in the mobile ecosystem. For the purpose of this paper, I define the app ecosystem complexity as having two aspects: the number of apps of all categories and the number of apps in each category for a mobile platform. The rationale behind the twofold definition is that the categorization of apps is arbitrary. Regardless of definition, some app categories are made up of more apps 
than other categories are. For example, as of June 2014, almost 20\% of the apps (i.e., more than 200,000 apps) on the Apple's App Store belong to the "games" category $[18,74]$. To restate the previous argument, the more complex an ecosystem is (i.e., as the number of the apps either in total or within a category increases, and hence as the level of redundancy goes up), the more the ecosystem becomes reliable (i.e., the probability for an ecosystem to provide a consistent level of performance increases).

\section{Ecosystem Failure}

In reliability engineering theory, failure is defined as the probability that a system or a machine will not perform or underperform its intended function during a specified period of time under a given set of conditions [38]. In other words, failure denotes a state where a system is not successful to meet the demand for its services or products (See Figure 1).

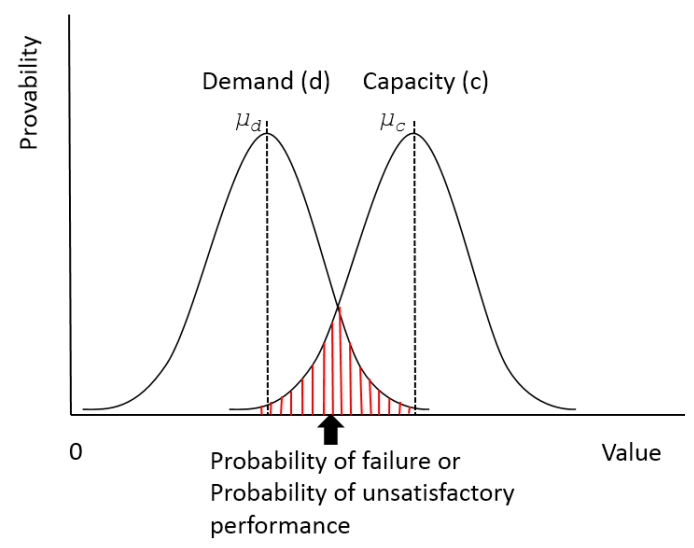

Figure 1. The probability of failure or unsatisfactory performance is shown as an area where demand exceeds capacity.

If there is a car, for example, at each demand for a service (i.e., transportation) the car may provide the service (i.e., success) or it may not (i.e., failure) depending on the status of the, among others, engine. As implied before, reliability is a probability of a system not to be failed. Reliability of a car, therefore, is the probability that the car will meet the demand during a specified period of time under a given set of conditions. Demand for services or products is often continuous, which means repeated demands over small units of time $\Delta t$ [31]. For example, a car may need to serve a series of demands (e.g., commute, shopping, dining out, giving a ride to someone, etc.). That is, let $p$ be the probability of failure upon demand and $r$ be the probability of success, or $1-p$, then reliability after $n$ demands $\left(R_{n}\right)$ is

$R_{n}=r^{n}$

and reliability after time $t$, which is the sum of $n$ units of $\Delta t$, or $t=n \Delta t$, is

$R(t) \cong e^{\lambda(t)}$

where $\lambda=p / \Delta t$ and $\Delta t$ is a small increment of time [42]. Therefore, reliability of a system that is in continuous service or operation without repair declines as time elapses.

Even without citing Winston Churchill, success is not final and failure is seldom total. For example, failure of a car does not mean that all parts of the car have failed but that the car as a whole effectively failed because it no longer provides the intended or expected service demanded of it. From an ecosystem (either a natural or a business ecosystem and its subsystems such as an app ecosystem) perspective, an ecosystem fails when it stops providing the services or products demanded of it. As with cars, the ecosystem in general does not have to fall down, although an ecosystem, particularly a business ecosystem, could collapse [1] or become part of a bigger ecosystem [50]. For example, if a mobile platform moves away from a desirable state as a thriving platform toward an undesirable state as a declined platform, the mobile ecosystem experiences a local diminution. It is still a functioning ecosystem though, but it has failed in the sense that it no longer develops markets as it might have used to and hence manifests unsatisfactory performance [17]. 


\section{Complexity and System Failure}

A system is a set of interacting or interdependent components forming an integrated whole [75]. As it gets complex, the number of components and their interdependency increases [82]. If each component in a system has a finite and independent probability of failure, and all components are mutually dependent upon each other for the functioning of a system, then the more complex a system (i.e., the more components it has) the more it will be prone to fail or the less reliable it will be. Mathematically, this is equivalent to

$R_{T}=\prod_{i=1}^{M} R i$,

where $R_{T}$ is the reliability of the system during a specified period of time, $R_{i}$ is the reliability of the $i^{\text {th }}$ component, $M$ is the number of components. If the reliability of each component of a system is 1 , then the reliability of the system as a whole is 1 so that the $R_{T}$ falls between 0 and 1 , inclusive.

This formula indicates that the reliability of a system decreases as the number of its components and their mutual interdependency increases. The same is true for an ecosystem, whether it is a natural one or not. The more functional groups or the number of apps required for an ecosystem to meet the demand for services or products at particular levels, the less reliable it becomes [57]. In other words, for an app ecosystem to become functioning and reliable at a desired level, it requires more apps. As the number of apps grows, the number of functional groups would also grow. We see this phenomenon in mobile ecosystems. Android and iOS mobile ecosystems thrive, while Windows Phone and other mobile ecosystems suffer from the dearth of the number of apps available and the number of mobile devices in consumers' hands $[17,80]$.

\section{Singularity versus Redundancy}

In a natural ecosystem, species are phyletically singular (i.e., a species takes an evolutionary change in a single line of descent without branching just like versioning of an app in an app ecosystem). Species, however, are rarely singular from an ecosystem's point of view (again, whether the ecosystem is a natural one or not). For example, in a natural ecosystem all photoautotrophs (i.e., all organisms that make nutrients by photosynthesis) remove carbon from the air, and all decomposers (i.e., organisms that break down dead or decaying organisms) get energy and nutrients from dead organic matter [45]. The same is true for business ecosystems. Their members compete, cooperate, and are inspired by each other to survive and to make themselves better in terms of functionality, performance, attractiveness, acceptance, etc. [67]. Species (members for a business ecosystem) vary in their levels of contributions to ecosystem processes. For example, a successful mobile platform may inspire more than others in the whole mobile ecosystem but contributes less to net mobile ecosystem advance than other mobile platforms if its, say, openness is limited (e.g., Android vs. iOS platforms).

Like the variation in species richness [41], the variation in member richness within a mobile ecosystem or, in a narrow sense, in an app ecosystem can lead to variation in both the number of functional groups (e.g., games category) and the number of either driver or passenger members within and among functional groups [18, 74]. This suggests that the apparent redundancy of members is due to the fact that most changes in member composition are likely to involve members within functional groups rather than changes in the number of functional groups. App ecosystem functioning is likely to show significant changes in response to variation in member composition when such changes involve the addition of functional groups. For example, we have recently witnessed the mushrooming of health-, fitness- and wearable-related apps and APIs [19, 21]. It can, therefore, be argued that an app ecosystem is likely to show significant reaction to variation in member composition when members with functional groups are dominated by a few members with new and/or innovative ideas (that is, at the burgeoning stage of a functional group). Thus, an ecosystem redundancy can formally be defined as

$C_{j}\left(P M_{j}\right)=C_{j}\left(P M_{j}-1\right)+\varepsilon=C_{j}\left(P M_{j}-2\right)+\varepsilon=\ldots C_{j}(1)+\varepsilon$,

where $C_{j}$ is the total contribution by any measure to the app ecosystem functioning by the $j^{\text {th }}$ functional group over a certain period of time $t$ as a function of $P M_{j}$, the number of passenger members in the $j^{\text {th }}$ functional group, and $\varepsilon$ is an error term resulted from the stochastic deviation from the mean app ecosystem function expected at that member richness. Upon holding the formula true, then the passenger members within the functional group are redundant. That is, $C_{j}$ is independent of $P M_{j}$. Consequently, the app ecosystem flows and hence its functioning will be affected by the member richness within a functional group, as well as the number of functional groups that are added as potent new 
apps arrive (For an app ecosystem the potential number of functional groups or categories appears limitless). A reason for this could be found from the monopolistically competitive app markets [13,68], as well as compensatory abilities of members within functional groups.

In a natural ecosystem, species redundancy is a result of the compensatory abilities of the species within functional groups [72]. Local diminution of species within functional groups is often followed by compensatory advance of others, which effectively leads to a replacement of the contributions of diminished species to overall functional group functioning. The same holds true for the members of a business ecosystem [57,62]. For example, not all the 200,000 game apps are successful; most of them just sit on app stores [18] [74]. Such compensatory redundancy is called parallel redundancy in engineering [42]. Parallel redundancy increases the reliability of an ecosystem, while singularity decreases the reliability.

\section{APP REDUNDANCY AND APP ECOSYSTEM RELIABILITY}

To formally describe the role of apps (members) in app ecosystem functioning, assume that the $i^{\text {th }}$ app within the $j^{\text {th }}$ functional group has a finite probability of local diminution $p_{i j}$, and the probability of the app being successful (or of being possible to provide contribution to app ecosystem functioning) over a period of time is equal to $\left(1-p_{i j}\right)$. Following the inference for the reliability of a system formulated by [42], the $P_{i j}$ over a certain increment in time $t$ is

$P_{i j}(t)=e^{-\lambda t}$,

where $\lambda=p_{i j} / \Delta t$ and $P_{i j}$ is the probability that the $i^{\text {th }}$ app in the $j^{\text {th }}$ functional group becomes successful after $t$. The locally diminished can become successful by calibration efforts such as improving the performance of them. If the probability of successful calibration of the $i^{\text {th }}$ app in the $j^{\text {th }}$ functional group equals $q_{i j}$, then the probability of unsuccessful calibration is $\left(1-q_{i j}\right)$, and following the derivation for reliability as above

$S_{i j}(t)=1-e^{-v t}$,

where $S_{i j}$ is the probability that the $i^{\text {th }}$ app in the $j^{\text {th }}$ functional group successfully recalibrates itself after t, and $v=q_{i j}$ IUt. As an app ecosystem to which the app belongs becomes more unsuitable or incompetent, $v \rightarrow 0$.

According to probability theory, if $R_{i j}$ is the probability for the $i^{\text {th }}$ app in the $j^{\text {th }}$ functional group to be successful by time $t, R_{i j}(t)$ is equal to the probability that diminution, calibration, or both occur, that is, $P\{P U S\}$, or

$R_{i j}(t)=P_{i j}(t)+S_{i j}(t)-P_{i j}(t) S_{i j}(t)$.

Figure 2 depicts this relationship for $v>0$ and $\lambda>0$. As long as $v>0$ over a long period of time, an app has a probability of greater than 0 of being successful at a certain point in time, even if $\lambda$ is much less than 1 (i.e., $\lambda<<1$ ). Functioning of a functional group depends on the number of apps within the group. If each app can compensate for the diminution of another by parallel redundancy, then the probability that a functional group will contribute to an app ecosystem is

$F_{j}(t)=1-\prod_{i=1}^{M j}[1-R i j(t)]$,

where $M_{j}$ is the total number of apps in the $j^{\text {th }}$ functional group. The probability for an app ecosystem to be functioning over time $t$ if it contains $N$ functional groups is

$G(t)=\prod_{j=1}^{N} F j(t)$. 


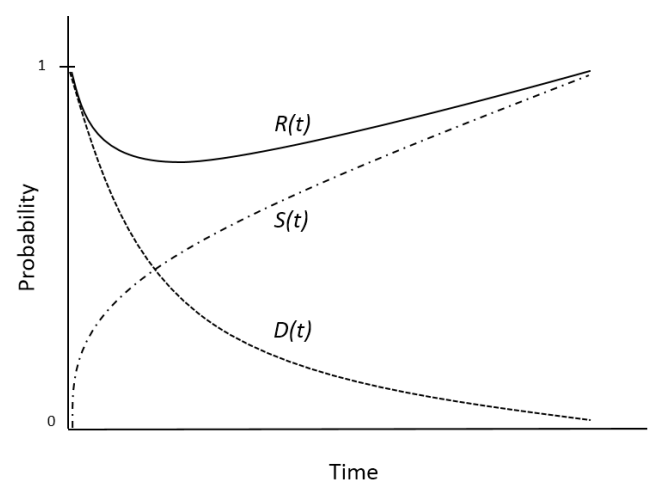

Figure 2. The probability for an app to become successful in an app ecosystem (R) as a function of the probability of local diminution (D) and calibration (S) over time when $v=0.005$ and $\lambda=0.005$.

It is apparent from the last three formulas that increasing app redundancy within app ecosystems increases the reliability of complex app ecosystems. Figure 3 shows how the level of reliability increases as both the number of apps and the number functional groups increase.
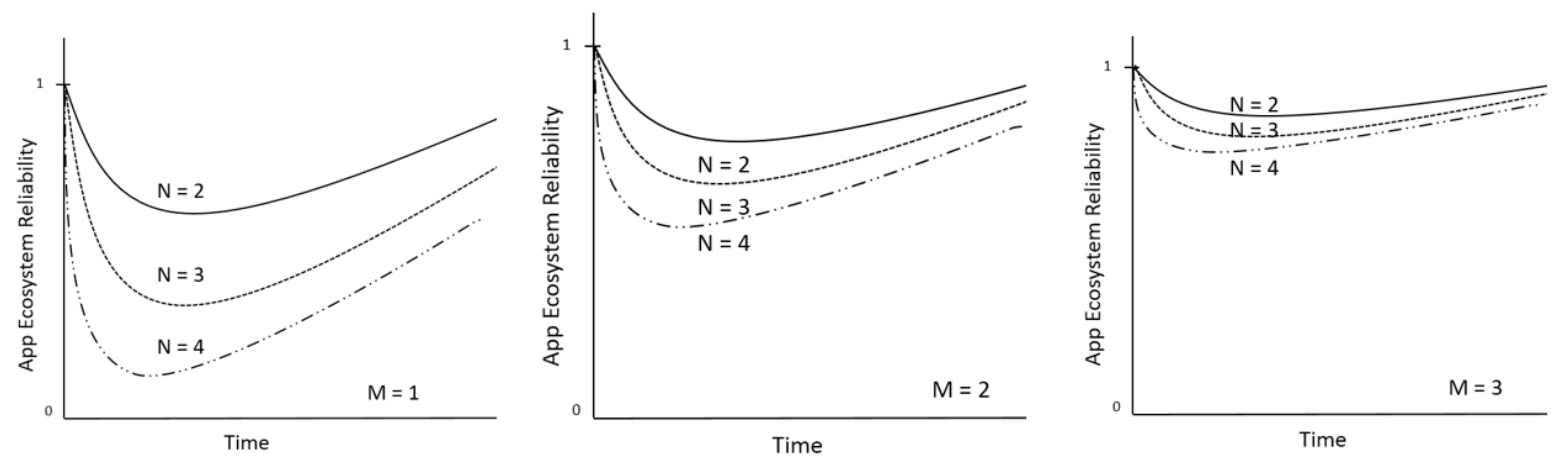

Figure 3. The relationship between app ecosystem reliability $\mathrm{G}(\mathrm{t})$, the number of functional groups $\mathrm{N}$, and the number of apps per functional group $\mathrm{M}$, when $v=0.005$ and $\lambda=0.005$. If $v=0$, all curves approach zero asymptotically.

In other words, increasingly complex ecosystems (i.e., as the value $\mathrm{N}$ increases) are seemingly less reliable (i.e., an app ecosystem appears to be less reliable when the number of apps and, consequently, the number of functional groups starts picking up at the burgeoning stage of it), but increasing app redundancy (i.e., as $\mathrm{M}$ increases, that is, the number of apps either in an ecosystem or in functional groups increases.) can compensate decrease in reliability. This implies that an app ecosystem is likely to be functioning (i.e., providing services or products upon demand) over long period of time as long as the number of apps increases, but they are not globally (i.e., across app ecosystems) declined or even extinct and their recalibration is possible.

These models rely on the assumptions that constancy and independency of app diminution and calibration probabilities. In an app ecosystem neither diminution nor calibration rates are constant (e.g., calibration may be an annual event depending on the modification routines [59]) or independent (e.g., better calibration may rescue diminution of an app). Relaxing these assumptions and developing corresponding models is a straightforward process for systems in which probability for component failure is neither independent nor constant [42]. But even new models based on the relaxed assumptions would not change qualitative conclusions from the models described above.

\section{VALUE OF APP REDUNDANCY}

Since the introduction of iPhone in 2007, the growth of smartphones and apps has been astonishing. According to [16], there are about 160 million smartphone users in the United States. By the end of 2014, the number of mobile subscriptions will reach 2.3 billion worldwide [37]. One of the main forces behind this phenomenal growth can be attributed to the explosive growth in the number of apps. This means a tremendous success to certain app ecosystems but does not necessary mean all the apps enjoy the same kind of success. With millions of apps already on the app 
stores, and tens of thousands more launched every month, many developers and startups are finding that their success is far from reality. Most of the apps face local diminution, being forgotten, or extinction within their ecosystems [25] [71]. Ecosystems, either a natural or a business, often contain many members per functional group. Local diminution of members within functional groups is unavoidable and frequent, but it could be transient if they are sustainable over a certain period of time via recalibration. In an app ecosystem, the possibility for passenger apps to be rescued and the possibility for driver apps to keep their success over a long period of time are very important because declining app diversity means loss of app redundancy in the app ecosystem. From the arguments presented in this paper, it appears almost inevitable that app richness within functional groups provides redundancy in app ecosystem functioning, a potentially important feature affecting app ecosystem reliability. In the absence of sudden, dramatic changes in the mobile ecosystem environment $[29,78]$, it is possible that app ecosystems will continue to function for a long period of time, but the reliability of their functioning will be dependent on the richness of their apps and the redundancy this richness gives to app ecosystems.

\section{REFERENCES}

1. Ando, R., \& Rigby, B. (2013). Microsoft swallows Nokia's phone business for $\$ 7.2$ billion. Reuters. Available: http://in.reuters.com/article/2013/09/03/microsoft-nokia-idINDEE98202R20130903).

2. Annenberg Learner. (n.d.) What factors determine extinction probability? Learner. Available: (http://www.learner.org/courses/biology/textbook/biodiv/biodiv_6.html).

3. Arvind, A., \& Hicks, J. 2006. A mobile phone ecosystem: MIT and Nokia's joint research venture. IEEE Intelligent Systems, 21(5), pp. 78-79.

4. Asay, M. 2013. Mobile developers find more opportunity, and money, in enterprise over consumer apps, ReadWrite. Available: (http://readwrite.com/2013/12/18/mobile-developers-enterprise).

5. Basole, R., \& Karla, J. 2011. On the evolution of mobile platform ecosystem structure and strategy. Business \& Information Systems Engineering 3(5), pp. 313-322.

6. Basole, R. 2009. Visualization of interfirm relations in a converging mobile ecosystem. Journal of Information Technology, 24, pp. 144-159.

7. Bender, G. M., Kot, L., Gehrke, J., \& Koch, C. 2013. Fine-grained disclosure control for app ecosystems, in Proceedings of the 2013 ACM SIGMOD International Conference on Management of Data, New York, NY, January 22-27, 2013 (869-880). New York: ACM.

8. Biodiversity. (n.d.) Biodiversitya-z.org. Available: http://terms.biodiversitya-z.org/terms/23

9. Brand, F. S., \& Jax, K. (2007). Focusing the meaning(s) of resilience: resilience as a descriptive concept and a boundary object. Ecology and Society (12:1), pp. 23.

10. Briscoe, G., \& De Wilde, P. (2006). Digital ecosystems: evolving service-orientated architectures, in Proceedings of the 1st international conference on Bio inspired models of network, information and computing systems, Cavalese, Italy, December 11-13, 2006 (Article No 17). New York: ACM.

11. Cadenasso, M. L., Pickett, S. T. A., \& Grove, J. M. (2006). Dimensions of ecosystem complexity: Heterogeneity, connectivity, and history. Ecological Complexity (3). pp. 1-12.

12. Campbell, N. A., \& Reece, J. B. (2004). Biology ( $7^{\text {th }}$ Ed.). Benjamin Cummings.

13. Chamberlin, E. H. (1962). The theory of monopolistic competition: A re-orientation of the theory of value ( $8^{\text {th }}$ Ed.). Harvard University Press.

14. Chang, E., \& West, M. 2006. Digital ecosystems a next generation of the collaborative environment, in Proceedings of the 8th International Conference on Information Integration and Web-based Applications \& Services, Yogyakarta, Indonesia, December 4-6, 2006 (3-23).

15. Clapham, A. R., Lucas, C. E., \& Pirie, N. V. (1976). A review of the United Kingdom contribution to the International Biological Programme. Philosophical Transactions of the Royal Society (B 274), pp. 277-555.

16. comScore. (2014). comScore Reports January 2014 U.S. Smartphone Subscriber Market Share. comScore. Available: https://www.comscore.com/Insights/Press-Releases/2014/3/comScore-Reports-January-2014-USSmartphone-Subscriber-Market-Share).

17. Connors, W., \& Sentana, M. (2014). BlackBerry losing traction in developing markets. The Wall Street Journal. Available: http://online.wsj.com/news/articles/SB10001424052702303636404579397114023735516).

18. Costello, S. (2014). How many apps are in the iPhone app store? About.com. Available: http://ipod.about.com/od/iphonesoftwareterms/qt/apps-in-app-store.htm). 
19. Danova, T. (2014, May 22). WEARABLE APPS: Inside the race to create a new app ecosystem. Business Insider. Available: http://www.businessinsider.com/wearable-apps-inside-the-race-to-create-a-new-app-ecosystem-20143).

20. Davis, F. D. (1989). Perceived usefulness, perceived ease of use, and user acceptance of information technology. MIS Quarterly 13(3), pp. 319-340.

21. Dredge, S. 2014. Health and fitness apps booming ahead of Apple's iOS 8 launch. The Guardian. Available: http://www.theguardian.com/technology/2014/jun/19/health-fitness-apps-apple-ios-8).

22. Eadicicco, L. 2014. A Google executive reveals why the company came up with its beautiful new Android design. Business Insider. Available: http://www.businessinsider.com/google-material-design-android-2014-7).

23. Ecosystem. (n.d.) Biology Online. Available: http://www.biology-online.org/dictionary/Ecosystem

24. Evolutionary Environment Habitat Network. (2007). Source Forge. Available: http://evenet.sourceforge.net

25. Freierman, S. (2011). One million mobile apps, and counting at a fast pace. The New York Times. Available: http://www.nytimes.com/2011/12/12/technology/one-million-apps-and-counting.html?_r=0).

26. Fried, I. (2014). Flappy bird flap continues as hard-to-master game pulled from App Store, Google Play. Re/code. Available: http://recode.net/2014/02/09/flappy-bird-flap-continues-as-hard-to-master-game-pulled-from-app-storegoogle-play

27. Gartner says less than 0.01 percent of consumer mobile apps will be considered a financial success by their developers through. (2018). Gartner. Available: http://www.gartner.com/newsroom/id/2648515

28. Gitay, H., Wilson, J. B., \& Lee, W. G. (1996). Species redundancy: A redundant concept? Journal of Ecology, 84, pp. 121-124.

29. Gustin, S. (2013). The fatal mistake that doomed BlackBerry. Time. Available: http://business.time.com/2013/09/24/the-fatal-mistake-that-doomed-blackberry

30. Hairston, N. G., Smith, E. F., \& Slobodkin, L. B. (1960). Community structure, population control, and competition. American Naturalist, (106). pp. 249-257.

31. Hanemann, W. M. (1984). Discrete/continuous models of consumer demand. Econometrica, 52(3), pp. 541-561.

32. Harte, J., \& Kinzig, A. P. (1993). Mutualism and competition between plants and decomposer: Implications for nutrient allocation in ecosystems. American Naturalist (141), pp. 829-846.

33. Henle, K., Sarre, S., \& Wiegand, K. (2004). The role of density regulation in extinction processes and population viability analysis. Biodiversity \& Conservation, 13(1). pp. 9-52.

34. Hill, M. O. (1973). Diversity and evenness: A unifying notation and its consequences. Ecology (54), pp. 427-432.

35. Iansiti, M., \& Levien, R. (2004). The Keystone advantage: What new dynamics of business ecosystems mean for strategy, innovation, and sustainability. Boston, MA: Harvard Business School Press.

36. Iansiti, M., \& Levien, R. (2004). Strategy as ecology. Harvard Business Review, 82(3), pp. 68-78.

37. ICT. (2014). The world in 2014. ICT Facts and Figures. ICT. Available: http://www.itu.int/en/ITUD/Statistics/Documents/facts/ICTFactsFigures2014-e.pdf

38. IEEE. (1991). IEEE standard computer dictionary. A compilation of IEEE standard computer glossaries, IEEE Available: http://ieeexplore.ieee.org/servlet/opac?punumber=2267

39. Jax, K. (2005). Function and "functioning" in ecology: What does it mean? Oikos (111), pp. 641-648.

40. Larizadeh, A. (2013). Eight tips for a successful app. Forbes. Available: http:/www.forbes.com/sites/avidlarizadeh/2013/07/19/eight-tips-for-a-successful-app

41. Lawton, J. H., \& Brown, V. K. (1994). Redundancy in ecosystems. Biodiversity and Ecosystem Function Springer Study Edition (99), pp. 255-270.

42. Lewis, E. E. (1995). Introduction to reliability engineering ( $2^{\text {nd }}$ Ed.). Wiley.

43. List of mobile software distribution platforms. n.d. Wikipedia. Available: http://en.wikipedia.org/wiki/List_of_mobile_software_distribution_platforms

44. Levin, S. (1998). Ecosystems and the biosphere as complex adaptive systems. Ecosystems (1), pp. $431-436$.

45. Loreau, M., Naeem, S., Inchausti, P., Bengtsson, J., Grime, J. P., Hector, A., Hooper, D. U., Huston, M. A., Raffaelli, D., Schmid, B., Tilman, D., \& Wardle, D. A. (2001). Biodiversity and ecosystem functioning: Current knowledge and future challenges. Science, 294(5543), pp. 804-808.

46. Lunden, I. (2013). Gartner: 102B app store downloads globally in 2013, \$26B in Sales, 17\% from in-app purchases. Gartner. Available: http://techcrunch.com/2013/09/19/gartner-102b-app-store-downloads-globally-in2013-26b-in-sales-17-from-in-app-purchases

47. May, R. M. (1972). Will a large complex system be stable? Nature, 238(5364), pp. 413-414.

48. McGahan, A. M. (2004). How industries change. Harvard Business Review, 82(10), pp. 86-94. 
49. McIntyre, D. A. (2014). 10 brands that will disappear in 2015: 24/7 Wall St. The Huffington Post. Available: http://www.huffingtonpost.com/2014/07/12/brands-disappearing-in2015_n_5580761.html?ir=Business\&utm_hp_ref=business

50. Microsoft. (2011). Nokia and Microsoft announce plans for a broad strategic partnership to build a new global mobile ecosystem. Press release Available: http://www.microsoft.com/en-us/news/press/2011/feb11/0211 partnership.aspx

51. Millennium Ecosystem Assessment (MA). (2005). Ecosystems and human well-being: General synthesis. Available: http://www.unep.org/maweb/documents/document.356.aspx.pdf

52. Mobile App Categories. n.d. Google. Available: https://developers.google.com/adwords/api/docs/appendix/mobileappcategories

53. Montoya, D. (2008). Habitat loss, dispersal, and the probability of extinction of tree species. Communicative \& Integrative Biology, 1(2), pp. 146-147.

54. Moore, J. F. (1996). The death of competition: Leadership and strategy in the age of business ecosystems. New York: Harper Collins.

55. Moore, J. F. (2006). Business ecosystems and the view from the firm. Antitrust Bulletin, 51(1), pp. 1-58.

56. Mora, C., Danovaro, R., \& Loreau, M. (2014). Alternative hypotheses to explain why biodiversity-ecosystem functioning relationships are concave-up in some natural ecosystems but concave-down in manipulative experiments. Scientific Reports (4), doi:10.1038/srep05427.

57. Mueller, D. C. (1972). A life cycle theory of the firm. The Journal of Industrial Economics, 20(3), pp. $199-219$.

58. Naeem, S., \& Li, S. (1997). Biodiversity enhances ecosystem reliability. Nature (390), pp. 507-509, doi: $10.1038 / 37348$.

59. Nelson, R. R., \& Winter, S. G. (1982). An evolutionary theory of economic change, Cambridge: Harvard University Press.

60. Nguyen, L. A. (2014). Exclusive: Flappy bird creator dong Nguyen says app 'Gone forever' because it was 'An addictive product.' Forbes. Available: http://www.forbes.com/sites/lananhnguyen/2014/02/11/exclusive-flappybird-creator-dong-nguyen-says-app-gone-forever-because-it-was-an-addictive-product

61. Odum, E. P. (1953). Fundamentals of ecology, Philadelphia, PA: W.B. Saunders.

62. Osawa, J. (2014). What Snapchat can learn from Asian messaging apps. The Wall Street Journal. Available: http://blogs.wsj.com/digits/2014/08/21/what-snapchat-can-learn-from-asian-messaging-apps

63. Ovaskainen, O., \& Meerson, B. (2010). Stochastic models of population extinction. Arxiv.org. Available: http://arxiv.org/ftp/arxiv/papers/1008/1008.1162.pdf

64. Peltoniemi, M. (2006). Preliminary theoretical framework for the study of business ecosystems. Emergence: Complexity \& Organisation, 8(1), pp. 10-19.

65. Petsas, T., Papadogiannakis, A., Polychronakis, M., Markatos, E. P., \& Karagiannis, T. (2013). Rise of the planet of the apps: A systematic study of the mobile app ecosystem, in Proceedings of the 2013 Conference on Internet Measurement Conference, Barcelona, Spain, October 23-25, 2013 (277-290). New York: ACM.

66. Phillips, T. (2014). Apple, Google now rejecting Flappy Bird clones, reports suggest. Eurogamer. Available: http://www.eurogamer.net/articles/2014-02-17-apple-google-now-rejecting-flappy-bird-clones-reports-suggest

67. Porter, M. (1980). Competitive strategy: Techniques for analyzing industries and competitors. New York: The Free Press.

68. Rajiv, G., \& Rahul, T. (2013). Inferring app demand from publically available data. MIS Quarterly 27(4), pp. 1253-1264.

69. Root, R. B. (1967). The niche exploitation pattern of the blue-gray gnatcatcher. Ecological Monographs (37), pp. 317-350.

70. Rothschild, M. (1990). Bionomics: Economy as ecosystem, New York: Henry Holt and Company.

71. Rubin, B. F. (2013). The dirty secret of apps: Many go bust. The Wall Street Journal. Available: http://online.wsj.com/news/articles/SB10001424127887324582804578346221047028366

72. Sala, O. E., Lauenroth, W. K., McNaughton, S. J., Rusch, G., \& Zhang, X. (1996). Functional role of biodiversity: a global perspective, H. A. Mooney, J. H. Cushman, E. Medina (Eds.), Wiley.

73. Schulze, E. D., Beck, E., \& Müller-Hohenstein, K. (1995). Plant Ecology, New York, NY: Springer-Verlag.

74. Statista. (2014). Most popular Apple App Store categories in June 2014, by share of available apps. Statista. Available: http://www.statista.com/statistics/270291/popular-categories-in-the-app-store

75. System. (n.d.) Merriam-Webster Online. Available: http://www.merriam-webster.com/dictionary/system).

76. Tansley, A. G. (1935). The use and abuse of vegetational concepts and terms. Ecology (16), pp. $284-307$. 
77. The 8 mobile app metrics that matter. (2014). Localytics. Available: http://www.localytics.com/blog/2014/the-8mobile-app-metrics-that-matter

78. The decline and fall of Nokia. (n.d.). Wikipedia. Available: http://en.wikipedia.org/wiki/ The_Decline_and Fall _ of Nokia

79. Tiwana, A., Konsynski, B., \& Bush, A. A. (2010.) Platform evolution: Coevolution of platform architecture, governance, and environmental dynamics. Information Systems Research, 21(4), pp. 675-687.

80. Tofel, K. C. (2014). Should Microsoft be worried about Windows phone's falling market share? Gigaom. Available: http:/gigaom.com/2014/08/15/should-microsoft-be-worried-about-windows-phones-falling-marketshare

81. Tuomisto, H. (2010). A consistent terminology for quantifying species diversity? Yes, it does exist. Oecologia (4), pp. 853-860.

82. Von Bertalanffy, L. (1969). General system theory: Foundations, development, applications/ Penguin University Books.

83. Walker, B. H. (1992). Biodiversity and ecological redundancy. Conservation Biology (6), pp. 18-23.

84. Walker, B. H. (1995). Conserving biological diversity through ecosystem resilience. Conservation Biology (9), pp. 747-752.

85. Wardle, D. A., \& Zackrisson, O. (2005). Effects of species and functional group loss on island ecosystem properties. Nature, I, pp. 806-810. doi:10.1038/nature03611.

86. Willis, A. J. (1997). The ecosystem: An evolving concept viewed historically. Functional Ecology, 11(2), pp. 268271.

87. Wilson, J. B. (1999). Guilds, functional types and ecological groups. Oikos (86), pp. 507-522.

88. Wolfram, S. (1988). Complex system theory, in Emerging Syntheses in Science: Proceedings of the Founding Workshops of the Santa Fe Institute, 183-189, Addison-Wesley.

89. Zio, E. (2009). Reliability engineering: Old problems and new challenges. Reliability Engineering \& System Safety 94(2), pp. 125-141. 